\title{
Application of Model-Based Data Transmission Techniques to Gravitational Model Data
}

\author{
Jeremy Straub \\ Department of Computer Science, University of North Dakota, Grand Forks, USA \\ Email: jeremy.straub@my.und.edu
}

Received June 12, 2013; revised July 19, 2013; accepted August 11, 2013

Copyright (C) 2013 Jeremy Straub. This is an open access article distributed under the Creative Commons Attribution License, which permits unrestricted use, distribution, and reproduction in any medium, provided the original work is properly cited.

\begin{abstract}
The transmission of scientific data over long distances is required to enable interplanetary science expeditions. Current approaches include transmitting all collected data or transmitting low resolution data to enable ground controller review and selection of data for transmission. Model-based data transmission (MBDT) seeks to increase the amount of knowledge conveyed per unit of data transmitted by comparing high-resolution data collected in situ to a pre-existing (or potentially co-transmitted) model. This paper describes the application of MBDT to gravitational data and characterizes its utility and performance. This is performed by applying the MBDT technique to a selection of gravitational data previously collected for the Earth and comparing the transmission requirements to the level required for raw data transmission and non-application-aware compression. Levels of transmission reduction up to $31.8 \%$ (without the use maximum-error-thresholding) and up to $97.17 \%$ (with the use of maximum-error-thresholding) resulted. These levels significantly exceed what is possible with non-application-aware compression.
\end{abstract}

Keywords: Spacecraft Communications; Link Budget Reduction; Gravitational Model Data; Gravitational Model Data Processing; High-Value Transmission; Deep Space Enabling Technologies

\section{Introduction}

Deep space planetary science missions and those venturing beyond the solar system are met with a significant challenge. As a spacecraft journeys away from the Earth, the power, antenna size and pointing accuracy required, to transmit a given amount of data in a given amount of time, increases significantly. While larger spacecraft or larger, more powerful ground stations are potential solutions to this problem, they are not optimal. The transmission of raw data, while desirable from a reuse and scientific validity perspective, does not maximize the scientific return for most mission types.

Model-Based Transmission Reduction (MBTR) seeks to do this. MBTR reduces data throughput via the creation of a higher-value data product. Model-Based Data Transmission (MBDT) is the lowest level of MBTR: a building block for the higher levels. With MBDT, data of a known level of quality (a maximum discrepancy from the believed-true value) is transmitted more compactly via identifying required updates to a pre-existing or cotransmitted model. Analysis is performed on board the spacecraft to ensure that the most effective data transmission mechanism is utilized (use of existing model, co-transmission or raw data transmission), based on that particular data being prepared for transmission at the time.

This paper presents the MBTR methodology and reviews its value. It then presents, in detail, the algorithm for performing MBDT on gravitational model data and compares this to the use of MBDT for other data types. Next, data related to the Earth's gravitational model is processed using MBDT and results are presented an analyzed. Finally, the utility for this technology on board an interplanetary spacecraft is considered.

\section{Background}

This paper represents the fusion of four existing areas of research, which will be discussed in this section. These include space mission design, Model-Based Transmission Reduction, MBTR's Model-Based Data Transmission component (which has previously been implemented and demonstrated with image data) and gravitational modeling (and its associated data products). MBDT for gravitation modeling combines the MBTR-component, MBDT, to meet spacecraft communications design needs for the transmission of gravitational data. 


\subsection{Space Mission Needs}

Space mission design is the art of balancing competing needs for limited resources while achieving mission objectives and operating within mission constraints. For most spacecraft, budget, volume, mass and schedule are key constraints [1]. These primary constraints drive secondary constraints including power limitations (limited by generation capacity, generally a function of volume) and link budget (limited by power, frequency and antenna size) restrictions.

Wertz, et al. [1] present a twelve stage mission design process which flows high-level objectives through requirements and down to system-level design. This process is iterative and relies on mission designer ingenuity and flexibility to create a successful design. Others [2,3] have proposed similar approaches, with alternate steporders and different areas of focus. All, however, share the immersive approach to mission design.

Communication and onboard processing are key elements of this mission design process. Communications capabilities and window availability limits the amount of data that can be received from the spacecraft, and when it can be received. A variety of approaches for trading on board processing time for reduced a communication needs have been considered [eg, 4-7]. Historically, these could be subdivided into three categories: loss-causing compression, lossless compression and removal of extraneous information. Loss-causing compression can produce a significant reduction in data transmission requirements; however, this is at the expense of quality. Lossless compression is not able to achieve a high level of compaction that loss-causing compression can; however, is suitable for numerous applications that cannot tolerate the data loss and artificating caused by losscausing compression. The removal of extraneous information, generally, provides a transmission requirement reduction benefit commensurate with the amount of information removed. This category can be very beneficial, for certain applications; however, is not easily generalizable. All of these techniques require onboard computing resources to be consumed. This requires mass and volume to be devoted to these subsystems, as well as the use of power, heat dissipation and other supporting subsystem capabilities.

\subsection{Model-Based Transmission Reduction}

The MBTR paradigm has been presented previously $[8,9]$. It is a four level approach to reducing the amount of data that is required to transmit a given amount of knowledge. Each progressively higher level incorporates those below it. Each level, thus, requires progressively more onboard processing capability and produces a data stream with progressively higher value-per-byte. These four levels are Model-Based Data Transmission (MBDT), Model-Based Data Analysis (MBDA), Model-Based Result Transmission (MBRT) and Model-Based Findings Transmission (MBFT).

MBDT, the application of which to gravitational data is the subject of this paper, is the lowest level of MBTR. With MBDT, a low-resolution data set is utilized as a model for which a set of updates are created that will bring it to the higher-resolution level. Ideally, this model is pre-shared; however, in some circumstances, it may be effective to co-transmit the model with the update data. For example, this model may be data remotely sensed from Earth, prior to mission departure. MBDT is discussed in greater detail in Section 2.3.

MBDA enhances MBDT by incorporating a new context-aware heuristic for prioritizing data transmission. While MBDT data might have been prioritized based upon the magnitude of the variation of the model from the in-situ collected data, MBDA considers the importance of the variation, in light of pre-defined heuristics created based on applicable mission objectives. For example, this may include looking for regions of anomaly and prioritizing these over single large anomalies (which might be attributable to sensor issues) or looking for certain patterns of anomaly and prioritizing these.

MBRT considers the scientific thesis that underlies the mission and prioritizing data for transmission. This includes considering whether the data provides support or refutation for each applicable thesis being tested by the mission, prioritizing the data based upon the thesis that supports or refutes and by the level of support for refutation offered by the data. Consideration may also be given, particularly, to data in a class with limited membership (e.g., support for thesis without other support).

The top level of MBTR, which provides the highest value-level Data Products, is MBFT. Under NBFT, the model of the phenomenon of interest is updated to reflect conclusions supported by collected data. Assertions made based upon this revised model are transmitted to controllers, along with data that supports the assertions. This allows controller validation of the proper performance of the onboard analysis software.

While MBTR offers a significant benefit to planetary science missions, this is not without cost. This cost is comprised of two main areas: the requirement for increased onboard processing capabilities (and associated supporting subsystems) and the loss of raw data for secondary analysis or validation. This would be particle a problematic if it was later discovered that the onboard software was flawed in some way, and its conclusions cannot be trusted. This risk can be partially mitigated by causing the spacecraft to retain data for as long as possible (until the storage is needed for another use), such that updated software could be used to reprocess the data on- 
board. Data supporting particularly important conclusions might be stored indefinitely (until the end of the spacecraft's useful life).

In [10], it was shown that the MBTR paradigm MFT technique could be combined with a robotic control architecture to produce a multi-robot collaborative data collection framework. This framework utilizes data fusion techniques to combine data from multiple heterogeneous craft with different modes of movement and rangelevels.

\subsection{Model-Based Data Transmission}

MBDT is a technique for reducing data transmission requirements. In $[9,11]$, MBDT was demonstrated to decrease the file size of image data. It was shown that MBDT outperformed common image compression techniques both in cases where a pre-shared model was used and in cases where co-transmission of the model was utilized. Unlike most image compression techniques (e.g., JPEG) a maximum-possible level of error (and thus a guaranteed level of quality) was known and could be relied upon. It was also shown that MBDT could be utilized, when updates were co-transmitted with JPEG (or other) compressed image data to create this guaranteed maximum level of error. While not considered in [9] or [11], lossless compression could also be implemented to compress the model update data, further decreasing the transmission requirements.

MBDT for image data begins with the acquisition of high-resolution imaging. This is compared to the lowerresolution model (created experimentally by reducing the resolution of the image file) on a pixel-by-pixel basis. A difference a value for each pixel is obtained (in [11], black and white imagery was processed). These values are compared to the minimum guaranteed quality (MGQ) threshold value. Those exceeding the MGQ are included in a set of update messages for transmission back to controllers. A low-overhead format for these messages has been implemented. The update file's size is compared to that required to transmit the raw data, as a model that is particularly inaccurate (or misaligned) may require more data transmission to correct than the actual data set. The creation of a co-transmitted model can also be considered, as in some cases is more efficient to update the lowresolution model and then supply changes then to provide changes to a model of limited accuracy. For larger data sets, model updates could be performed on a regional basis, instead of a global one.

\subsection{Gravitational Model Data}

Gravitational field data has a multitude of uses in planetary science. This data reflects the local density of the body, which intensifies are weakens the local gravitational pull. Density data can be used to differentiate be- tween material types and potentially identify body composition and structure.

\subsubsection{Use on Earth}

Gravitational modeling is used extensively on Earth. It can be utilized to locate basins of ground water, by those seeking petroleum, by those that are exploring for natural resources, to detect geological faults and other hazards or to detect the internal structure of volcanos [12].

Niu, et al. [13] demonstrate one use for remote-sensed gravitational field data. They compare gravitational data from the Gravity Recovery and Climate Experiment (GRACE) to their Simple Groundwater Model (SIMGM) which is used as part of a global climate model (GCM). From this work, they determined that groundwater recharge creates a more-wet soil moisture profile and between $4 \%$ and $16 \%$ more evaporation than gravitational free drainage. They attribute this greater evapotranspiration to evaporation from the soil's surface because it is wetter.

Tapley, et al. [14] presents findings derived from gravity modeling performed by the Gravity Recovery and Climate Experiment (GRACE) spacecraft, which further demonstrate the utility of a gravity model for understanding water flow, on a global scale. In this instance, a model of known phenomenon was compared to the data collected by GRACE to identify additional factors that needed to be accounted for. Tapley, et al. proffer that water differences represented the largest source of model and collected-data disparity. This water storage data, the note, is useful in predicting a plethora of phenomena including climate change, flooding, weather patterns and the productivity of agricultural land. The GRACE data was able to identify very small water-level changes which effected large bodies of water. The largest variation was the Amazon basin which varied between 7.7 $\mathrm{mm}$ below the average value to $14 \mathrm{~mm}$ above it. The data, they note, had a level of error between 2 and $3 \mathrm{~mm}$, for features of approximately $600 \mathrm{~km}$ in size in 2003 and $1000 \mathrm{~km}$ in 2002 (with accuracy increasing due to a software revision loaded on to the spacecraft). They note that they found a largely cyclical pattern on a year-toyear basis with some variations (e.g., the region containing Africa's tropical rainforests was found to be drier in 2003 than in 2002). This analysis requires an understanding between the interactions of numerous systems including the atmosphere and ocean. Furthermore, random-error-attributable effects must be excluded.

Johnson, et al. [15] discuss their work related to characterizing the Seattle fault in Washington. This fault was originally identified in 1965 based on the detection of gravitational data. As late as 1991, additional gravitational data was still being utilized to extend the believed-scope of the fault (into Elliot Bay). Gravitational mapping-based work interpreted the fault as a single con- 
tinuous structure; however, Johnson et al.'s work, based on seismic reflection, suggests that the fault is more complex. This, thus, demonstrates the utility of gravitational mapping for fault detection and its limited granularity (requiring refinement from the fusion of in-situ sensed data, in some cases).

Kauahikaua, et al. [16] proffer that their analysis of gravitational data has "yielded an unprecedented view of the substructure of each volcano making up the island" of Hawai'i. Through this analysis, they believe that they are gaining a better understanding of how pathways for magma formed during the process of the formation of Hawai' $i$ and that future work, based on this data, may help to understand how seismic energy may be released and specific causes of large-scale landslides. Kauahikaua, et al. collected 3300 gravitational measurements over a $1400 \times 1400 \mathrm{~km}$ area to produce a $250 \times 250 \mathrm{~km}$ model of the island, corrected for the effects of the downward-warping of the oceanic crust in the region. They corrected for known topographic and oceanic features and produced a 3-d model of the region. Analysis compared a base density of $2300 \mathrm{~kg} / \mathrm{m}^{3}$, assigned to abovewater terrain, and $2600 \mathrm{~kg} / \mathrm{m}^{3}$, assigned to below-water terrain, to the higher density $\left(2900 \mathrm{~kg} / \mathrm{m}^{3}\right)$ typical of molten magma and basaltic intrusions. They found the average density of the island to be approximately 2820 $\mathrm{kg} / \mathrm{m}^{3}$. They proffer that this analysis has helped to understanding the plumbing of the island's volcanos and the island's geologic history.

\subsubsection{Use beyond Earth}

In [17], an outline for a near-Earth asteroid (NEA) intervention mission is presented. NEA intervention can utilize several approaches (outlined in [18]). Intervention strategies include slowly shifting the NEA off of its Earth-impacting trajectory (either utilizing a NEA-attached propulsion device or gravitational pull of a spacecraft in close proximity), deploying a nuclear charge to change the momentum and/or break apart the NEA or causing a kinetic impact to change its trajectory. For most of these approaches, and to select between them, characterization of the material composition of the NEA is required. Additionally, the composition of the NEA must be known to determine how to best implement intervention strategies which involve close-proximity or surface operations.

Iess, et al. [19] assess possible structural and material compositions for Saturn's largest moon, Titan, using gravitational field data collected by the Cassini spacecraft. Of Cassini's over fifty fly-bys of Titan, four were devoted to gravity field characterization. The gravity field was measured via tracking the Doppler shift in the microwave-band transmission from the spacecraft to the ground. The gravity field was determined via tracking the spacecraft's range rate data, which was measured to a level of accuracy of $7.5 \times 10^{-5}$ meters per second. From this data Iess, et al. theorize about several possible configurations of Titan. They proffer that Titan likely is comprised of an ice-layer which surrounds a rock core. They note that there may be an intermediate layer of ice-rock mixture. They indicate that this data may refute a previous claim by Zebker, et al. [20], who presumed that Titan may have frozen at a different altitude from Saturn than it currently occupies. Iess, et al. note, contradicting this, that Titan's gravity field closely resembles that which would be expected from a fluid body.

Smith, et al. [21] combine gravitational and laser altimeter data collected by the Mars Global Surveyor spacecraft to create a model of the seasonal variation of ice cover on Mars. During the warmer period, much of this material is in the atmosphere; however, it condenses back onto the surface of the planet when the temperature cools. This, Smith, et al. proffer results in an redistribution of 1/20,000,000 of the planet's mass on an annual basis. Using the combined gravitational and laser altimeter data, they are able to characterize the density of the ice formation to $910 \pm 230 \mathrm{~kg} / \mathrm{m}^{3}$. This, it is noted, is a higher density than ice on the Earth, despite Mars having a lesser gravitational pull to compact it. They proffer that this is an expected result, given the composition of the $\mathrm{CO} 2$ and dust (representing $10 \pm 40 \%$ of the deposited mass) ice.

Folkner, et al. [22] seek to determine the polar moment of inertia for the planet Mars. They proffer that this value will improve the accuracy of internal composition models of the planet (constraining the solution set of possible models) and help to ascertain the amount of mass that is transferred between the polar ice caps and the atmosphere. Data from surface-based measurement as well as Doppler shift data from the Mars Pathfinder and Viking missions has been utilized to determine a set of projected values, which are presented by Folkner, et al. Subsequent work has further refined these numbers.

Zuber, et al. [23] utilize the same gravitational and laser altimeter data collected by the Mars Global Surveyor spacecraft as [21] to characterize the internal structure of Mars and project its early history. By comparing the altimeter data with the altimeter data, Bouguer gravity anomalies are identified. These anomalies indicate differences in crust thickness and density and the presence of various features (e.g., volcanos). Based on this analysis, Zuber, et al. proffer that the crust of Mars has an average thickness of $50 \mathrm{~km}$ and a maximum and minimum thickness of $92 \mathrm{~km}$ and $3 \mathrm{~km}$, respectively. They also note that the density is projected to be $2900 \mathrm{~kg} / \mathrm{m}^{3}$. In addition to generating this basic statistical data about Mars, the fusion of the two data sources allows a projecttion about water flows on Mars earlier in the planet's history. Underground channels, projected to be about 200 $\mathrm{km}$ in width with lengths of thousands of kilometers, are 
projected to have transported water (in significant quantity) and sediment to northern areas. This sediment is predicted to have had a role in the northern lowland resurfacing.

\subsubsection{Data Product Utilized for Testing}

The work described herein utilizes the EGM2008 Global Gravitational Model dataset [24] created by the National Geospatial-Intelligence Agency. This data product [25] contains 233,301,600 geoid undulations covering the Earth as a 1-meter by 1-meter grid (equi-angularly spaced) in 10,801 rows and 21,600 columns. The data values range from -106.910 to 85.840 . For display purposes, these have been normalized on a 0 to 255 range to produce a greyscale image. Figure 1 shows a visual depiction of a partial area $(5000 \mathrm{~m} \times 5000 \mathrm{~m})$ of the Earth's gravitational model. Figure 2 depicts the gravitational model for the entire Earth. Note that this display is based on the EGM2008 grid, which skews the actual orientation of the Earth somewhat (note the fact that most continents appear at an unnatural angle, for example).

\section{Model Based Data Transmission of Gravitational Model Data}

MBDT of gravitational data builds from previous work $[9,11]$ on reducing data transmission requirements for image data through the comparison of source data to a pre-shared or co-transmitted model. The highest level of reduction is possible with a pre-shared model. For planetary science work (e.g., a mission for data collection to another planet) this model could be generated from Earth-based or Earth-orbiting (e.g., a satellite) observa-

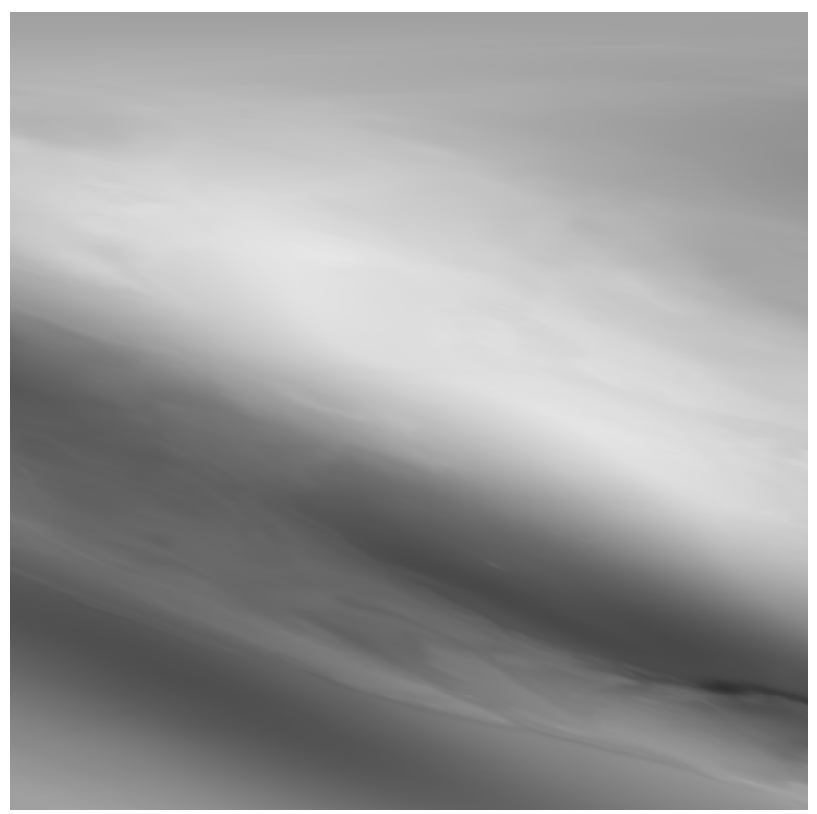

Figure 1. Earth's gravitational model, $5000 \times 5000$ meter area, produced from [24].

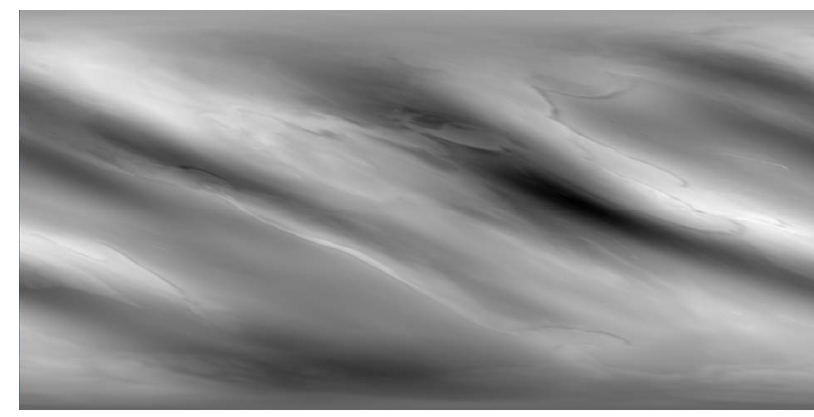

Figure 2. Earth gravitational model, produced from [24].

tions. This allows the base model, which may be resolution-limited by the sensitivity of the instrumentation, to be transmitted over high speed data links. Updates, which should (presuming accuracy of the initial model, subject to resolution limitations) be smaller can then be transmitted over the lower-bandwidth, higher-cost data link between the spacecraft in orbit of the remote planet.

Some gravity model data is collected via monitoring spacecraft telemetry from Earth. The projected orbit is compared to the actual orbit and discrepancies are utilized to identify gravitational features of the planet. In this instance, the gravity model is assembled on Earth, and no transmission (and thus transmission bandwidth reduction technique) is applicable. To attain the highest possible data, however, multiple orbital craft have been deployed. GRACE [14] demonstrated this technique in Earth orbit; GRAIL [26] demonstrated this same approach for gravity mapping of the moon.

For Earth observation purposes, the data link is not as large of an issue for pristine-class spacecraft (e.g., typical large missions like GRACE); it may still be for small spacecraft (such as those utilizing SmallSat and CubeSat form factors). The MBTR technique can be used, for these missions, to prioritize data for transmission, based on the size of the discrepancy from the model and to create a data product with a known maximum level of error, without having to incur the costs to transmit and store a high resolution model. This may be particularly important for ad hoc users of the data (e.g., users in the field).

A basic algorithm implements the process of determining what the most effective way to transmit the data is. Figure $\mathbf{3}$ depicts this MBDT decision-making algorithm for gravitational data. It makes the decision as to whether to transmit no data, model-updates, the model combined with updates or the raw data based on the target resolution, the correlation of the higher-resolution data with the model and the communications bandwidth available.

\subsection{Transmission with Pre-Existing Model}

MBDT update messages are utilized to make minor corrections to a model. If the model is pre-shared (e.g., col- 


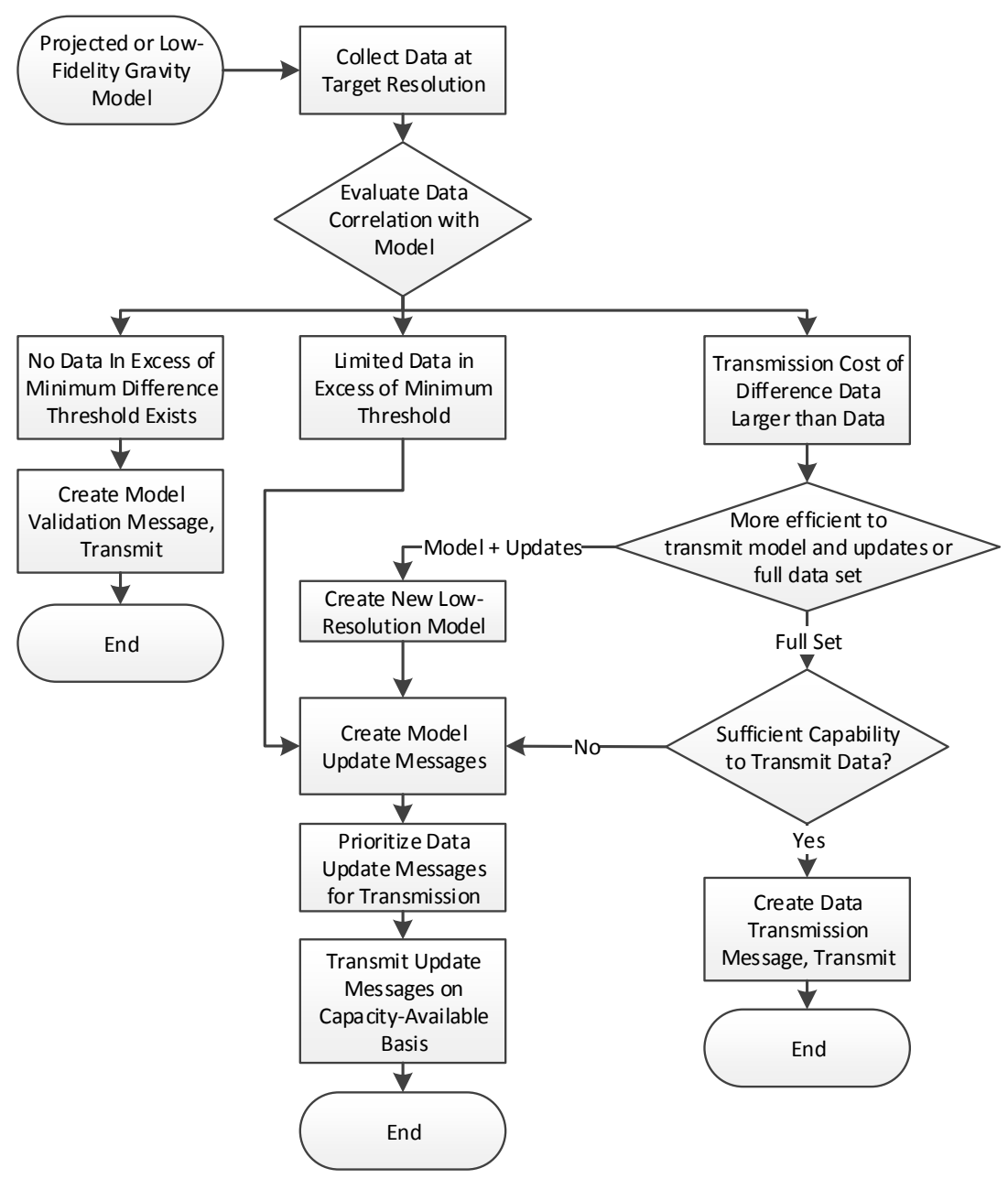

Figure 3. MBDT decision model for gravitational data processing.

lected on Earth and pre-loaded onto the spacecraft before launch), then only updates are required to be sent. Model updates, conceptually, can be either single-value or region-based. The work described herein only utilizes single-value updates, as the model is well-aligned (due to being derived from the high-resolution source data that it will be compared to). Region-based updates are used to correct a large area of data that is significantly different than the model to allow this to be performed as one large bounded update that can be overridden by one or more single-value updates.

\subsection{Co-Transmission with Model}

If a model is not available to be pre-shared or the model turns out to be highly inaccurate, an alternate (but not as efficient) MBDT process can be utilized to minimize transmission requirements. In this instance, a low-resolution model is created based on the target-resolution data and is transmitted with the update messages. The updates are then applied to the model at the destination. In some cases, this will require less data transmission than transmitting the raw data. Both the low-resolution model and the update messages can, generally, benefit from similar compression techniques as could be applied directly to the raw data, and thus outperform the approach of simply compressing the model. As shown in Figure 3, the efficacy of using this approach to reduce transmission requirements is validated before transmission (to ensure that transmitting the raw data wouldn't be more efficient).

\subsection{Maximum Error Thresholds}

In many cases, small errors in data are unimportant to a given application. For example, a small deviation in a graphic file may not create a perceptible (or application-relevant) difference to the image. A small deviation in a topographic data file will, in many cases, have no impact on the routing of an aircraft. While these small errors may have no relevance to a particular application, they can create a significant number of update messages. A maximum error threshold approach ignores error levels below the threshold by not transmitting update messages 
for them. This work implements a maximum grid-location-difference approach. However, thresholds can be implemented on a regional as well as local basis (corresponding to the triggering or suppression of regional and local updates).

\section{Data Format}

The data format, for gravitational data, is derived from the format used for image data in $[9,11]$. As with this prior work, the update message includes a message header, a section header and section data. The same data segmentation method is utilized; this avoids requiring several bytes of location information for each grid location change (which would be required if all changes were defined in a global-to-image context). Due to the utilization of the same format, interoperability between different data types is possible. This is important for higher levels of MBTR which require the analysis of multiple data types in order to derive and validate high-value conclusions.

\subsection{Header}

The header format is exactly the same as used in $[9,11]$. The header consists of five fields: craft identification, transmission identification, sequence number, time/date stamp and validation.

The craft identification field is a locally unique value that identifies the source craft. This field is utilized for identifying the collecting (or processing) craft when one craft relays for another. Data products produced via processing and analysis of data from other craft stamp the message with their own ID. Validation data (allowing ground controllers to verify correct functionality of the processing routines) retains the craft identifier of its collecting craft.

The transmission identification field has two key purposes: it identifies the transmission (uniquely to an implementation) and it identifies the data type and target of the data. The format of this field is not strongly defined, allowing it to be utilized in the most effective manner for each implementation (based on the number of expected targets, transmissions and data types).

The sequence number field is used to identify the order of messages that relate to a given target (based on a common transmission identification field value). This field is particularly important if area-level changes are made, as the order of application (e.g., the area is applied first and then the individual values) is important.

The time/date stamp field is utilized to store the date and time that data is collected at or a processed data product is produced. The validation field, which is optional, stores a checksum or hash value that can be utilized to ensure that no changes (e.g., due to transmission errors or otherwise) have affected the message. Many lower-level protocols will provide this service, rendering this field unneeded in those instances.

\subsection{Section Header}

The section header identifies the location of the section within the data set. Each section is $256 \times 256$ grid coordinates. This allows single-byte $(28 \times 28=65,536$ grid locations) to be used for each of the $\mathrm{X}$ and $\mathrm{Y}$ coordinates within the section, decreasing file size. The section header includes its own $\mathrm{X}$ and $\mathrm{Y}$ location, within the larger data set. These values are stored in 5-byte fields. A 2-byte field is used to identify the length of the section.

\subsection{Section Data}

The section data format is data type-specific; however, the format used for gravitational data is structurally similar to the format utilized for image data in $[9,11]$. Like with image data, two prospective addressing schemes can be utilized. The first includes a coordinate set ( $x$ and $y$ ) for each correction. The alternate, which is preferable if it is believed that lines will have an average of one or more corrections, includes an entry for each line in the data set. Lines are delimited with a set of all zeros (an x-coordinate of 0 , correction value $=$ all zeros $)$.

\subsection{Encapsulation into Lower-Level Data Format}

Neither MBTR or its MBDT component defines lowlevel transmission procedures. In terms of the OSI Model (see [27]), the entire MBTR process (including the MBDT work described herein) operates at the application layer (layer 1). However, it must make use of lower-level services (layers 2 - 7) to transmit data, as required. The protocol utilized will vary by application. For space applications, protocols such as the Space Data Link Protocol (CCSDS 132.0-B-1) [28] and the Space Packet Protocol (CCSDS 133.0-B-1) [29] are recommended standards. MBTR and MBDT are also useful for aerial and surface robotics applications; applications in these mediums will (particularly if not communicating with space assets, as discussed in [10]) generally utilize alternate communications protocols.

\section{Experimental Design}

A five-phase experiment has been conducted to validate the efficacy of MBDT approach for use with gravitational model data. These phases include 1) measuring the transmission requirements for a selection of the raw data; 2) measuring the transmission requirements when ZIP (DEFLATE/RFC 1951 [30]) compression is utilized; 3) applying and measuring transmission requirements when the MBTR approach is utilized; 4) applying and measuring transmission requirements when the MBTR approach 
incorporating a maximum-error threshold is utilized; and 5) applying and measuring transmission requirements when the maximum-error threshold MBTR approach is combined with ZIP compression.

\subsection{Transmission Requirements for a Selection of Raw Data}

The first phase of the experiment, the control condition, will involve characterizing the data transmission requirements for the raw data. A $5000 \times 5000$ grid location section of data will be selected from the global model, shown in Figure 2. The size of this file is compared to the data generated in phases two through five.

\subsection{Transmission Requirements with ZIP Compression}

The second phase of the experiment tests a simple approach to reducing transmission requirements: compression. In this instance, the common ZIP format is utilized with DEFLATE/RFC 1951 [30] compression. Numerous compression techniques, which do not require or rely on knowledge of the underlying data, exist. Their comparison is beyond the scope of this work. It is important to note, however, that any compression technique will involve a trade-off between onboard resources required for compression and decreased transmission requirements. Thus, the selection of a particular compression technique requires a knowledge of the computer system (e.g., the onboard computing system of a spacecraft or UAV) that it will be running on. Comparing computational requirements for MBDT versus conventional compression techniques represents a potential focus for future work.

\subsection{Implementing the MBDT Approach}

Phase three tests the MBDT approach to reducing gravitational data transmission requirements. In this phase, the pre-shared model and model-plus-updates approaches (described in Sections 3.1 and 3.2) are compared, with multiple model resolution levels (of 5\%,10\%, 25\% and $50 \%$ of the high-resolution version). These eight experimental conditions are compared with each other in terms of the resulting size of the data to be transmitted. Note that all of these approaches will result, when the updates are integrated back into the model, with the same high-resolution data as the original.

\subsection{The MBTR Minimum-Error Threshold Approach}

As discussed in Section 3.3, in many cases limited error is acceptable. Phase four tests the value of accepting different levels of error in further reducing transmission requirements. Maximum acceptable error (MAE) thresholds, the maximum amount of difference allowable (rep- resented as a percentage of the total range of possible data) between the high-resolution imagery and the result of combining the model with a set of updates are thus incorporated. MAE levels of 5\%, 10\% and $25 \%$ are tested in conjunction with model resolutions of $5 \%, 10 \%$, $25 \%$ and $50 \%$ of the high-resolution data. Data is collected for both pre-shared and co-transmitted model approaches.

Comparing the resulting transmission requirements across these 24 experimental conditions is, however, not valid, as the data products are of substantially different levels of quality. In [11] a metric for combining quality and file size, image quality as a function of file size (IQFFS), was utilized. A similar metric will be utilized for gravitational data: data accuracy as a function of file size (DAFFS). This metric is defined and named in such a way as to be applicable to numerous other applications. IQFFS is, thus, now a special image-data-only case of DAFFS. The DAFFS metric is derived by dividing the one minus the average grid location difference value (AGLDV) by the file size. In the image MBDT work presented in [11], the file size was represented in $1 / 10$ $\mathrm{MB}$ units, so as to keep the numbers within a convenient range. It is expected that DAFFS metric value units will vary from application to application and experiment to experiment, based on the type and size of data that is being processed.

\section{Results}

This section presents the results of the experiments described in Section 5. This begins with documenting the size of the base data and the reduction possible with ZIP-style file compression. Then the MBDT approach is implemented initially without and subsequently with the incorporation of a MAE threshold.

\subsection{Transmission Requirements for a Selection of Raw Data}

The base data used in this experiment consumes 65,536 bytes of data. This data is depicted in Figure 4.

\subsection{ZIP Compression Data}

Application of ZIP compression to the entire Earth-covering gravitational model provides a $3 \%$ decrease in file size.

\subsection{MBDT Approach Data}

Low-resolution models were created at four different sizes, comparative to the resolution of the base data: $5 \%$, $10 \%, 25 \%$ and $50 \%$. Note that the model resolution levels are percentages of the height and width (e.g., 5\% means $5 \%$ of the base height and $5 \%$ of the base width), not of the total area. Table 1 presents the sizes of these 
models. Table 2 presents the model size as a percentage of the base data, for comparison purposes. Note, from Table 2, that the models are larger than the comparative surface area, due to file format constraints. Figures 5(a)(d) display the models for comparison.

The models that were created (and for which size data was presented in Tables 1 and 2) were then processed, comparative to the original, to generate a set of MBDT updates that, when applied, would make the model exactly the same as the original. Table 3 presents the size of the models (repeated from Table 1, for ease of comparison) and updates. It also presents the combined size of the model plus the applicable updates. Table 4 presents this data as a percentage of the base data size.

\subsection{MBDT Minimum-Error Threshold Approach Data}

MBDT was next applied with MAE thresholds which specified a level of error that was acceptable (meaning

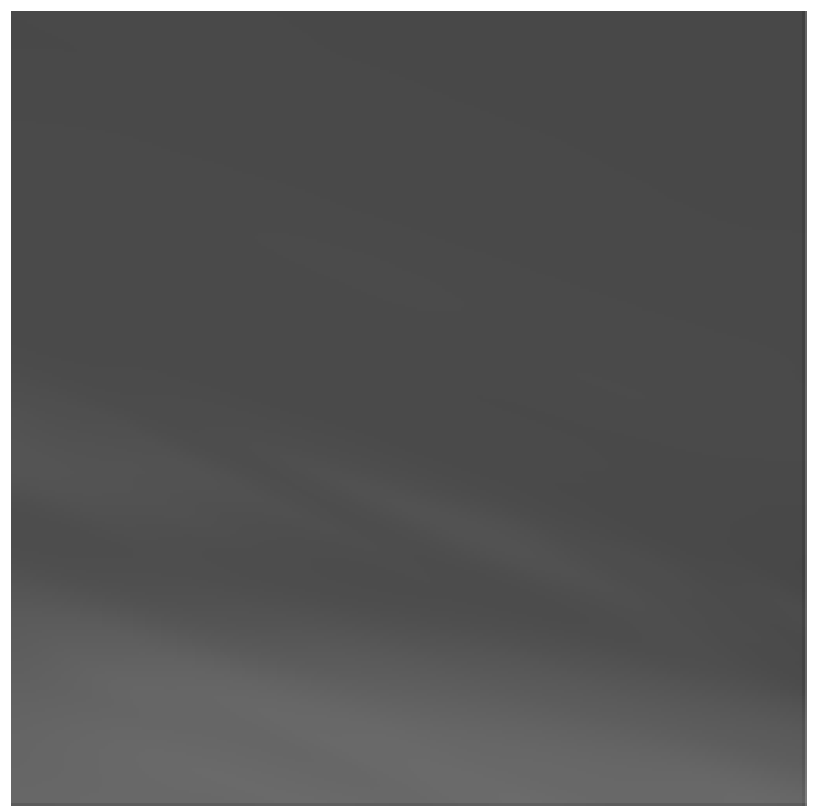

Figure 4. Visual depiction of raw data file used for experimentation. (a)

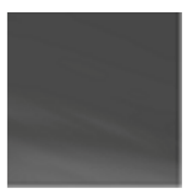

(c)

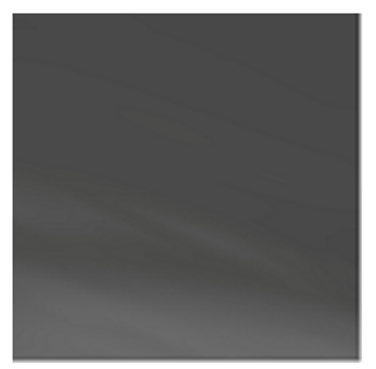

(d)
Figure 5. (a) 5\% model, left; (b) $10 \%$ model, middle left; (c) $\mathbf{2 5 \%}$ model; middle right; (d) $\mathbf{5 0 \%}$ model, right. that for error at this level or below, no update would be generated). Table 5 presents the size of the MBDT updates for each model resolution level. Table 6 presents this data as a percentage of the base data.

In some cases, the model may be of insufficient quality to facilitate effective updating (e.g., the updates would be bigger than transmitting the raw data), in others no a priori shared model may exist. In this case, the model is transmitted with the updates. Table 7 presents the size of the base model combined with the updates required to correct it to the MAE threshold. Note that when a location is corrected, it is corrected to the exact value, not to a value that is within the MAE range (as there is no performance benefit for inexactness). Table 8 presents these values as a percentage of the base data.

The data with updates to MAE specifications, while not being of the same quality as the original, is of known-bounded error. This data may be usable for many

Table 1. Model size.

\begin{tabular}{ccccc}
\hline & \multicolumn{4}{c}{ Model Resolution Levels } \\
\cline { 2 - 5 } & $5 \%$ & $10 \%$ & $25 \%$ & $50 \%$ \\
\hline Model & 574 & 2134 & 12,342 & 49,206 \\
\hline
\end{tabular}

Table 2. Model size as a percent of base data.

\begin{tabular}{ccccc}
\hline & \multicolumn{4}{c}{ Model Resolution Levels } \\
\cline { 2 - 5 } & $5 \%$ & $10 \%$ & $25 \%$ & $50 \%$ \\
\hline Model & $0.88 \%$ & $3.26 \%$ & $18.83 \%$ & $75.08 \%$ \\
\hline
\end{tabular}

Table 3. Updates without MAE threshold.

\begin{tabular}{cccccc}
\hline & & \multicolumn{4}{c}{ Model Resolution Levels } \\
\cline { 3 - 6 } & & $5 \%$ & $10 \%$ & $25 \%$ & $50 \%$ \\
\hline \multirow{2}{*}{$\boldsymbol{N}$} & Model & 574 & 2134 & 12,342 & 49,206 \\
& Updates & 72,314 & 49,392 & 25,800 & 15,250 \\
& Combined & 72,888 & 51,526 & 38,142 & 64,456 \\
\hline
\end{tabular}

Table 4. Model size and updates plus model as a percent of base data size.

\begin{tabular}{ccccc}
\hline & \multicolumn{4}{c}{ Model Resolution Levels } \\
\cline { 2 - 5 } & $5 \%$ & $10 \%$ & $25 \%$ & $50 \%$ \\
\hline Updates & $110.34 \%$ & $75.37 \%$ & $39.37 \%$ & $23.27 \%$ \\
Combined & $111.22 \%$ & $78.62 \%$ & $58.20 \%$ & $98.35 \%$ \\
\hline
\end{tabular}

Table 5. Updates with MAE threshold.

\begin{tabular}{|c|c|c|c|c|c|}
\hline & & \multicolumn{4}{|c|}{ Model Resolution Levels } \\
\hline & & $5 \%$ & $10 \%$ & $25 \%$ & $50 \%$ \\
\hline \multirow{3}{*}{$\sum_{\Sigma}^{\infty}$} & $5 \%$ & 1842 & 10,204 & 4320 & 2304 \\
\hline & $10 \%$ & 1278 & 1440 & 4320 & 2296 \\
\hline & $25 \%$ & 1278 & 1278 & 1296 & 2296 \\
\hline
\end{tabular}


Table 6. Updates as percent of base.

\begin{tabular}{cccccc}
\hline & & \multicolumn{4}{c}{ Model Resolution Levels } \\
\cline { 3 - 6 } & & $5 \%$ & $10 \%$ & $25 \%$ & $50 \%$ \\
\hline \multirow{2}{*}{$5 \%$} & $2.81 \%$ & $15.57 \%$ & $6.59 \%$ & $3.52 \%$ \\
& $5 \%$ & $1.95 \%$ & $2.20 \%$ & $6.59 \%$ & $3.50 \%$ \\
& $10 \%$ & $1.95 \%$ & $1.95 \%$ & $1.98 \%$ & $3.50 \%$ \\
\hline
\end{tabular}

Table 7. Updates plus model.

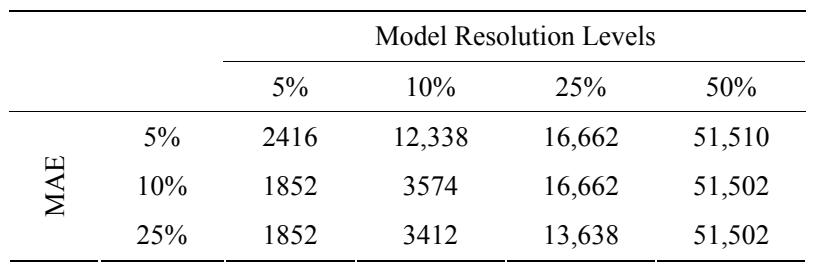

Table 8. Updates plus model as percent of base.

\begin{tabular}{|c|c|c|c|c|c|}
\hline & \multicolumn{4}{|c|}{ Model Resolution Levels } \\
\hline & & $5 \%$ & $10 \%$ & $25 \%$ & $50 \%$ \\
\hline \multirow{3}{*}{ 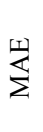 } & $5 \%$ & $3.69 \%$ & $18.83 \%$ & $25.42 \%$ & $78.60 \%$ \\
\hline & $10 \%$ & $2.83 \%$ & $5.45 \%$ & $25.42 \%$ & $78.59 \%$ \\
\hline & $25 \%$ & $2.83 \%$ & $5.21 \%$ & $20.81 \%$ & $78.59 \%$ \\
\hline
\end{tabular}

applications that can benefit from lower transmission requirements while suffering the applicable reduction in data quality. Figures 6-9 present data for the 5\% MAE threshold and the $25 \%$ MAE threshold with model sizes of $5 \%, 10 \%, 25 \%$ and $50 \%$.

\section{Analysis of Results}

The data presented in Section 6 demonstrates that ZIP compression is not an effective solution for compressing this format of gravitational data. While it is likely that additional compression (above 3\%) is possible with conventional compression techniques, this does not approach the level of transmission reduction possible with a format-aware reduction technique, such as MBDT.

MBDT results, without incorporating a MAE threshold, were able to reduce the data to $58.20 \%$ of its original size. This is a $31.8 \%$ reduction in file size, or about ten times the level of compression possible with the ZIP format. Incorporating the MAE threshold, MBDT was able to get the transmission to $2.83 \%$ of the base file or a $97.17 \%$ reduction in file size (allowing a MAE of up to $25 \%$, which may be too large to be suitable for many applications). With a MAE of 5\% (which would likely be suitable for most applications), the file size was reduced to $3.69 \%$ of base, or a $96.31 \%$ reduction.

It is notable, when looking at the update sizes in Tables 5 and 6 that the update sizes do not increase consistently with a smaller model. This is likely a characteristic of the data being relatively close in value. The data was

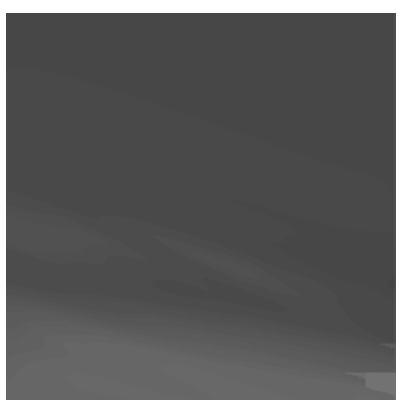

(a)

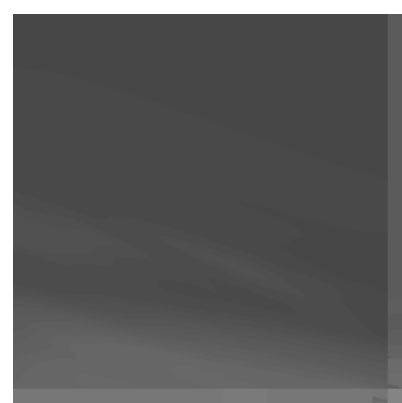

(b)
Figure 6. (a) $5 \%$ model with $5 \%$ MAE threshold, left; (b) $10 \%$ model with $5 \%$ MAE threshold, right.

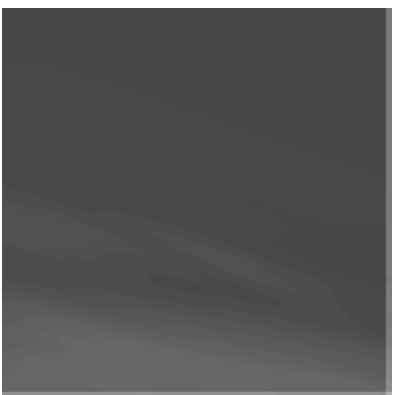

(a)

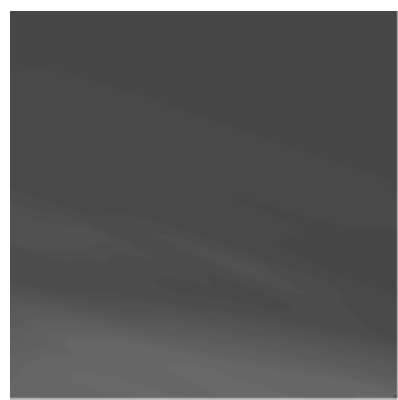

(b)
Figure 7. (a) $25 \%$ model with 5\% MAE threshold, left; (b) $50 \%$ model with $5 \%$ MAE threshold, right.

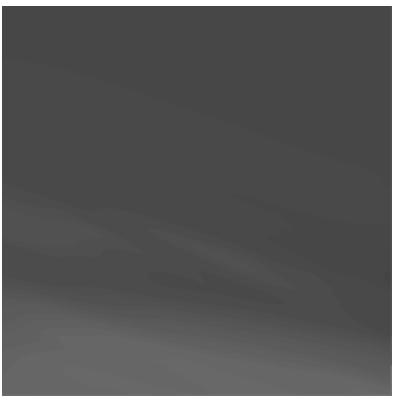

(a)

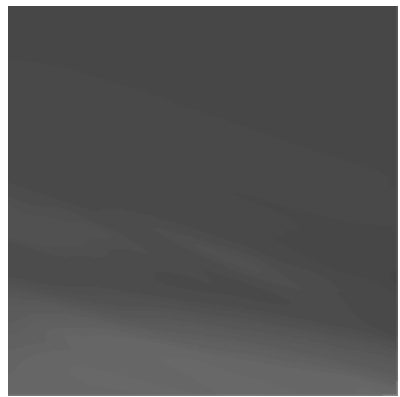

(b)
Figure 8. (a) 5\% model with 25\% MAE threshold, left; (b) $10 \%$ model with $25 \%$ MAE threshold, right.

intentionally selected to incorporate a noticeable variation (many areas, to a human viewer of its rendering appear simply to be a solid color or minor gradient). However, even with this variation, the difference is not dramatic across the image. This allows minor fluctuations to be dramatized by some size-reduction magnitudes, but not by others.

\section{Conclusion \& Future Work}

This work has demonstrated the viability of using the MBDT technique to reduce transmission requirements for gravitational model data. A pre-existing data set for the Earth has been utilized to demonstrate the approach's 


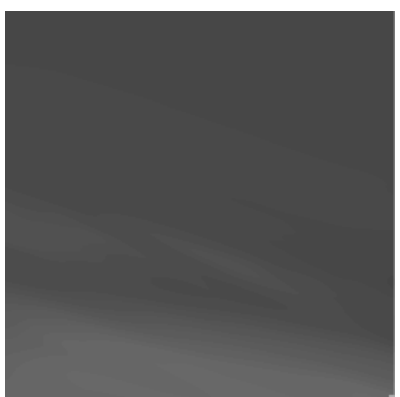

(a)

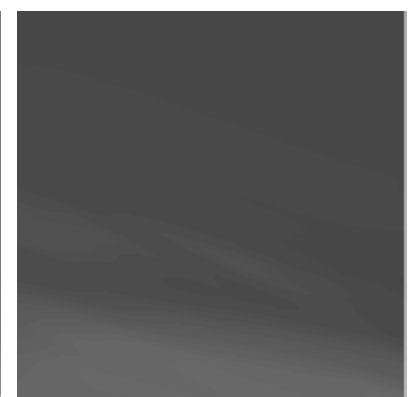

(b)
Figure 9. (a) 25\% model with 25\% MAE threshold, left; (b) $50 \%$ model with $25 \%$ MAE threshold, right.

efficacy; however, the techniques should be applicable to gravity models for other planets and space objects (e.g., asteroids, etc.). Future work will focus on applying MBDT to other gravitational data sets, applying MBDT to other data types and combining multiple data sources to generate higher-value data products as part of higher levels of the MBTR paradigm.

\section{Acknowledgements}

Small spacecraft development work at the University of North Dakota is or has been supported by the North Dakota Space Grant Consortium, the University of North Dakota Faculty Research Seed Money Committee, North Dakota NASA EPSCoR and the National Aeronautics and Space Administration.

\section{REFERENCES}

[1] J. R. Wertz, D. F. Everett and J. J. Puschell, "Space Mission Engineering: The New SMAD," 1st Edition, Microcosm Press, Hawthorne, 2011.

[2] P. Fortescue, G. Swinerd and J. Stark, "Spacecraft Systems Engineering," 4th Edition, John Wiley \& Sons, Inc., Hoboken, 2011. doi:10.1002/9781119971009

[3] W. J. Larson and J. R. Wertz, "Space Mission Analysis and Design," 3rd Edition, Microcosm Press, Hawthorne, 1999.

[4] J. Schou, P. H. Scherrer, R. I. Bush, R. Wachter, S. Couvidat, M. C. Rabello-Soares, R. S. Bogart, J. T. Hoeksema, Y. Liu, T. L. Duvall, D. J. Akin, B. A. Allard, J. W. Miles, R. Rairden, R. A. Shine, T. D. Tarbell, A. M. Title, C. J. Wolfson, D. F. Elmore, A. A. Norton and S. Tomczyk, "Design and Ground Calibration of the Helioseismic and Magnetic Imager (HMI) Instrument on the Solar Dynamics Observatory (SDO)," Solar Physics, Vol. 275, No. 1-2, 2012, pp. 229-259. doi:10.1007/s11207-011-9842-2

[5] L. N. Faria, L. M. G. Fonseca and M. H. M. Costa, "Performance Evaluation of Data Compression Systems Applied to Satellite Imagery," Journal of Electrical and Computer Engineering, Vol. 2012, 2012, Article ID: 471857.

[6] D. Barret, L. Ravera, P. Bodin, C. Amoros, M. Boutelier,
J. Glorian, O. Godet, G. Orttner, K. Lacombe, R. Pons, D. Rambaud, P. Ramon, S. Ramchoun, J. Biffi, M. Belasic, R. Clédassou, D. Faye, B. Pouilloux, C. Motch, L. Michel, P. H. Lechner, A. Niculae, L. W. Strueder, G. Distratis, E. Kendziorra, A. Santangelo, C. Tenzer, H. Wende, J. Wilms, I. Kreykenbohm, C. Schmid, S. Paltani, F. Cadoux, C. Fiorini, L. Bombelli, M. Méndez and S. Mereghetti, "The High Time Resolution Spectrometer (HTRS) Aboard the International X-Ray Observatory (IXO)," Proceedings of the SPIE 2010, Vol. 7732, 2010.

[7] K. M. Cheung, M. Belongie and K. Tong, "End-to-End System Consideration of the Galileo Image Compression System,” TDA Progress Report 42-126, 1996.

[8] J. Straub, "Increasing Interplanetary CubeSat Mission Science Return with Model Based Transmission Reduction," 1st Annual Interplanetary CubeSat Workshop, Boston, 29-30 May 2012.

[9] J. Straub, "Reducing Link Budget Requirements with Model-Based Transmission Reduction Techniques," Proceedings of the 26th Annual AIAA/USU Conference on Small Satellites, Logan, 13-16 August 2012, 18 Pages.

[10] J. Straub, "Integrating Model-Based Transmission Reduction into a Multi-Tier Architecture," Proceedings of the 2013 IEEE Aerospace Conference, Big Sky, 2-9 March 2013, pp. 1-7.

[11] J. Straub, "Model Based Data Transmission: Analysis of Link Budget Requirement Reduction," Communications and Network, Vol. 4, No. 4, 2012, pp. 278-287.

[12] G. R. Keller, "Using and Understanding Gravity Data," 2012.

http://research.utep.edu/default.aspx?pagecontentmode $=1$ \&tabid $=38186$

[13] G. Niu, Z. Yang, R. E. Dickinson, L. E. Gulden and H. Su, "Development of a Simple Groundwater Model for Use in Climate Models and Evaluation with Gravity Recovery and Climate Experiment Data," Journal of Geophysical Re-search, Vol. 112, No. D7, 2007, Article ID: D07103. doi:10.1029/2006JD007522

[14] B. D. Tapley, S. Bettadpur, J. C. Ries, P. F. Thompson and M. M. Watkins, "Grace Measurements of Mass Variability in the Earth System," Science, Vol. 305, No. 5683, 2004, pp. 503-505. doi:10.1126/science. 1099192

[15] S. Y. Johnson, S. V. Dadisman, J. R. Childs and W. D. Stanley, "Active Tectonics of the Seattle Fault and Central Puget Sound, Washington-Implications for Earthquake Hazards," Geological Society of America Bulletin, Vol. 111, No. 7, 1999, pp. 1042-1053. doi:10.1130/0016-7606(1999) $111<1042: A T O T S F>2.3 . C$ $\mathrm{O} ; 2$

[16] J. Kauahikaua, T. Hildenbrand and M. Webring, "Deep Magmatic Structures of Hawaiian Volcanoes, Imaged by Three-Dimensional Gravity Models," Geology, Vol. 28, No. 10, 2000, pp. 883-886. doi:10.1130/0091-7613(2000)28<883:DMSOHV $>2.0 . \mathrm{CO}$ ;2

[17] J. Straub and R. A. Fevig, "Earth Impactors: Threat Analysis and Multistage Intervention Mission Architecture," Proceedings of SPIE Volume 8385: Sensors and Systems for Space Applications V, SPIE 2012 Defense, 
Security + Sensing, Baltimore, 7 May 2012.

[18] J. P. McVey and N. Melamed, "Survey of Potentially Hazardous Object Threat Negation Campaign Options," Acta Astronautica, 2012, In Press.

[19] L. Iess, N. J. Rappaport, R. A. Jacobson, P. Racioppa, D. J. Stevenson, P. Tortora, J. W. Armstrong and S. W. Asmar, "Gravity Field, Shape, and Moment of Inertia of Titan," Science, Vol. 327, No. 5971, 2010, pp. 1367-1369. doi: $10.1126 /$ science. 1182583

[20] H. A. Zebker, B. Stiles, S. Hensley, R. Lorenz, R. L. Kirk and J. Lunine, "Size and Shape of Saturn's Moon Titan," Science, Vol. 324, No. 5929, 2009, pp. 921-923. doi:10.1126/science. 1168905

[21] D. E. Smith and M. T. Zuber, "Neumann, G. A. Seasonal Variations of Snow Depth on Mars," Science, Vol. 294, No. 5549, 2001, pp. 2141-2146. doi:10.1126/science. 1066556

[22] W. M. Folkner, C. F. Woder, D. N. Yuan, E. M. Standish and R. A. Preston, "Interior Structure and Seasonal Mass Redistribution of Mars from Radio Tracking of Mars Pathfinder," Science, Vol. 278, No. 5344, 1997, pp. 17491751. doi:10.1126/science.278.5344.1749

[23] M. T. Zuber, S. C. Solomon, R. J. Phillips, D. E. Smith, G. L. Tyler, O. Aharonson, G. Balmino, W. B. Banerdt, J. W. Head, C. L. Johnson, F. G. Lemoine, P. J. McGovern, G. A. Neumann, D. D. Rowlands and S. Zhong, "Internal Structure and Early Thermal Evolution of Mars from
Mars Global Surveyor Topography and Gravity," Science, Vol. 287, No. 5459, 2000, pp. 1788-1793. doi:10.1126/science.287.5459.1788

[24] N. K. Palvis, S. A. Holmes, S. C. Kenyon and J. K. Factor, "An Earth Gravitational Model to Degree 2160: EGM2008," Proceedings of the 2008 General Assembly of the European Geosciences Union, Vienna, 13-18 April 2008, 37 Pages.

[25] "Description of Files Related to Using the EGM2008 Global Gravitational Model to Compute Geoid Undulations with Respect to WGS 84," 2012.

http://earth-info.nga.mil/GandG/wgs84/gravitymod/egm2 008/README_WGS84_2.pdf

[26] GRAIL, "Mission Overview," 2012. http://www.nasa.gov/mission_pages/grail/overview/index. html

[27] H. Zimmermann, "OSI Reference Model—The ISO Model of Architecture for Open Systems Interconnection," IEEE Transactions on Communications, Vol. 28, No. 4, 1980, pp. 425-432. doi:10.1109/TCOM.1980.1094702

[28] Space Data Link Protocol, 2012. http://public.ccsds.org/publications/archive/132x0b1c1.pdf

[29] Space Packet Protocol. http://public.ccsds.org/publications/archive/133x0b1c2.pdf

[30] DEFLATE Compressed Data Format Specification Version 1.3. http://www.ietf.org/rfc/rfc1951.txt 